\title{
Further evidence for aberrant prefrontal salience coding in schizophrenia
}

\author{
Henrik Walter ${ }^{1,2 *}$, Stephan Heckers ${ }^{3}$, Jan Kassubek ${ }^{4}$, Susanne Erk ${ }^{1,2}$, Karel Frasch $^{5}$ and Birgit Abler ${ }^{6}$ \\ Department of Psychiatry, Division of Medical Psychology, University of Bonn, Bonn, Germany \\ 2 Department of Psychiatry, Division of Mind and Brain Research, Charité University Medical Center, Campus Charité Mitte, Berlin, Germany \\ ${ }^{3}$ Department of Psychiatry, Vanderbilt University, Nashville, TN, USA \\ ${ }^{4}$ Department of Neurology, University of UIm, Ulm, Germany \\ ${ }^{5}$ Department of Psychiatry II, University of UIm, Günzburg, Germany \\ ${ }^{6}$ Department of Psychiatry III, University of UIm, UIm, Germany
}

\section{Edited by:}

Andreas Meyer-Lindenberg, Central Institute of Mental Health, Germany

\section{Reviewed by:}

Peter Kirsch, Zentralinstitut für

Seelische Gesundheit, Germany;

Christian Wolf,

Heinrich-Heine-Universität, Germany

*Correspondence:

Henrik Walter, Department of

Psychiatry, Divison of Medical

Psychology, Rheinische

Friedrich-Wilhelms-University Bonn

Sigmund-Freud-Str. 25, D-53105 Bonn,

Germany.

e-mail: henrik.walter@ukb.uni-bonn.de
The revised dopamine hypothesis of schizophrenia postulates that dopamine metabolism is impacted differently with increased dopamine in the subcortical mesolimbic system and decreased dopamine in prefrontal cortical regions. Recently, we described findings supporting this hypothesis using a financial reward task in patients with schizophrenia (Walter et al., 2009). In addition to analysing prediction and prediction error coding, we found in this study evidence for aberrant cortical representation of salience in the right ventrolateral prefrontal cortex (VLPFC) in patients. Here, we reanalysed data of four other published reward studies of our group in order to investigate (i) whether we could replicate this finding in an independent cohort of patients with schizophrenia and (ii) how dopaminergic modulation impacts on cortical salience representation. Our main result was that we could replicate the finding of aberrant salience coding in the right VLPFC in patients with schizophrenia. Furthermore, we found evidence that the degree of salience coding in this region was correlated inversely with negative symptoms (anhedonia). Results of dopaminergic modulation showed tentative evidence for an influence of dopaminergic stimulation, but were not conclusive. In summary, we conclude that the right VLPFC might play a crucial role in salience coding and is impaired in schizophrenia.

Keywords: functional magnetic resonance imaging, schizophrenia, reward, salience, dopamine

\section{INTRODUCTION}

The dopamine hypothesis of schizophrenia (Carlsson and Lindqvist, 1963; Carlsson et al., 2000) assumes a central role for dopamine in the development of psychotic symptoms based on the mechanisms of antipsychotic drug action and PET studies on dopamine release in patients with schizophrenia following amphetamine intake. Although other neurotransmitters play an important role in the course of the illness, dopamine is still one of the most important scopes of current research on the neurobiology of schizophrenia. In its revised form hyperactivation of the mesolimbic dopamine system via D2-receptors is suggested to explain positive symptoms like delusions or hallucinations, while hypoactivation of the mesocortical system via D1-receptors is suggested to explain negative symptoms including anhedonia and cognitive impairment (Guillin et al., 2007). This view has recently been reframed as the aberrant salience hypothesis (Kapur, 2003; Kapur et al., 2006), for similar approaches compare (Spitzer, 1997; Heinz, 2002). This hypothesis is based on the idea that the mediation of incentive salience is the central function of mesolimbic dopamine (Berridge and Robinson, 2003). The anhedonia hypothesis of dopamine function (Wise et al., 1978) suggests a link between mesolimbic-mesocortical hypoactivation and negative symptoms.

Investigating the reward system with functional magnetic resonance imaging (fMRI) has been demonstrated as a reliable way to study the physiological function of the mesolimbic-mesocortical dopamine system in healthy subjects (Knutson et al., 2001; Knutson and Cooper, 2005; Abler et al., 2006, 2007; Yacubian et al., 2006; Jensen et al., 2007). Therefore, such paradigms are well suited to test the dopamine hypothesis of schizophrenia indirectly by investigating mesolimbic-mesocortical activation during reward processing. Aberrant activation in the ventral striatum of patients with schizophrenia has been demonstrated for prediction of financial reward (Juckel et al., 2006b; Jensen et al., 2008) and aversive cues (Jensen et al., 2008) as well as for reward prediction error (Murray et al., 2008). Aberrant activation of prefrontal regions during the outcome period of reward paradigms in schizophrenia has been described for the medial prefrontal cortex (MPFC) (Schlagenhauf et al., 2009) as well as for the right dorsolateral prefrontal cortex (DLPFC) (Corlett et al., 2007).

A recent investigation of our group provided support for the revised version of the dopamine hypothesis in partially remitted patients diagnosed with schizophrenia treated with the atypical neuroleptic olanzapine (Walter et al., 2009). Using a monetary incentive delay task in fMRI, we found evidence for normal to elevated activation in the ventral striatum for the prediction error during outcomes of reward. Moreover, cortical regions, namely the anterior cingulate gyrus and the right ventrolateral prefrontal cortex (VLPFC) mediating attentional processes and action selection were found to be hypoactive during reward processing. Importantly, while the above cited studies on schizophrenia directed their focus on either reward expectation or outcome signals related to prediction error theory, the finding of dysfunctional activation of the right 
VLPFC was revealed by modelling reward salience (Walter et al., 2009), that is, by comparing the most positive and most negative outcomes vs. the most neutral events (compare Section "Methods" and Figure 1). In line with the aberrant salience hypothesis of schizophrenia salience was thus related to its relevance to future behaviour (Cooper and Knutson, 2008): the more salient a cue, the greater the chance a subject will need to adapt a future behavioural response. Crucially, this response might involve either approach or withdrawal; cues related to negative outcomes like danger, loss or a need for escape will hold as much salience as cues related to positive or rewarding outcomes.

As the finding of schizophrenia associated aberrant salience coding in the right VLPFC is new, we were interested in replication of our index study (study 1) (Walter et al., 2009). For this purpose we reanalyzed recently published data of an independent sample of patients with schizophrenia and healthy control subjects (study 2: replication study) (Abler et al., 2008). The first and primary aim was to find out if we could replicate the finding of aberrant salience coding in the right VLPFC in an independent cohort of patients with schizophrenia. Secondly, we investigated in both, the index and the replication study, if aberrant salience coding was related to psychopathological symptoms. We expected to find a putative hyperactivation of the ventral striatum to be related to positive symptoms and a putative hypoactivation of the prefrontal cortex to be related to negative symptoms. In order to investigate additional aspects of salience coding, we included a reanalysis of three further published reward studies of our group: a healthy control sample manipulating reward probability (study 3) (Abler et al., 2006), a study of restless legs syndrome (RLS)-patients on and off their dopaminergic medication (study 4, RLS-Study) (Abler et al.,

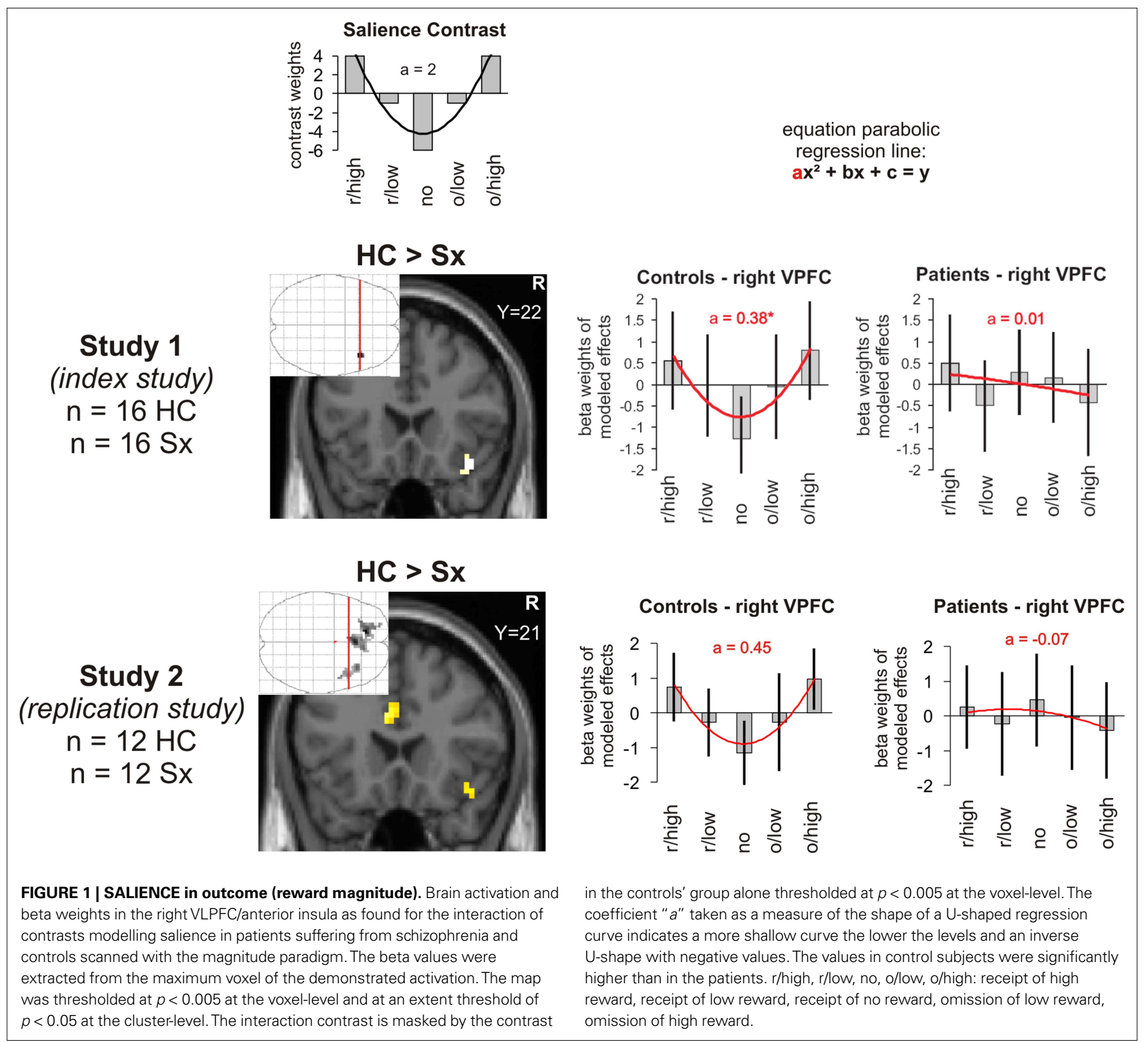


2009) and a study with healthy subjects receiving either placebo or a single dose of $5 \mathrm{mg}$ of olanzapine (study 5, olanzapine study) (Abler et al., 2007). We expected salience coding to be abolished by olanzapine in healthy controls and alteration of salience coding in RLS patients on their dopaminergic medication.

\section{MATERIALS AND METHODS}

Data from five studies published on monetary reward processing by our group (see Table 1) were reanalysed for salience coding during outcome. Studies 1 and 2 investigated partially remitted patients with schizophrenia medicated with atypical neuroleptics, study 3 healthy controls, study 4 patients with RLS on and 2-5 days (five half-lives of the respective drug) off their regular dopaminergic medication (pramiprexole, cabergoline, or ropinirole) and study 5 healthy controls receiving a single dose of either placebo or $5 \mathrm{mg}$ olanza- pine. Groups are characterised in detail in Table 1, more detailed information can be found in the respective publications. Within the respective studies, data were analysed during anticipation of reward as well as during outcome of reward including prediction error. The contrast modelling salience (compare Figures 1 and 2) had only been used in study 1 ("index study") but not in the other published studies and is reported here for the first time. Also the correlations with psychopathology are reported here for the first time.

\section{SUBJECTS}

Number, gender and age of the subjects investigated can be found in Table 1. None of the subjects had a history of major medical or neurological illness, besides RLS according to standardized diagnostic criteria in study 4 . None of the included healthy subjects or RLS patients was identified to have a history of psychiatric illness. Detailed

Table 1 | Studies (re)analyzed in this paper for the salience contrast

\begin{tabular}{|c|c|c|c|c|c|}
\hline & $\begin{array}{l}\text { Subjects } \\
\text { (Male/female) }\end{array}$ & $\begin{array}{l}\text { Age } \\
(\text { Mean } \pm S D)\end{array}$ & Medication & $\begin{array}{l}\text { Reward } \\
\text { paradigm }\end{array}$ & Reference \\
\hline Study 1 & $7 / 9 \mathrm{HC}$ & $38.0 \pm 9.0$ & Patients all medicated & Magnitude & Walter et al. (2009) \\
\hline (index study) & $8 / 8 \mathrm{Sx}$ & $33.0 \pm 10.2$ & with olanzapine & & \\
\hline Study 2 & $7 / 5 \mathrm{HC}$ & $36.2 \pm 11.2$ & Patients all medicated with & Magnitude & Abler et al. (2008) \\
\hline (replication study) & $5 / 7 \mathrm{Sx}$ & $36.7 \pm 7.8$ & various atypical neuroleptics & & \\
\hline $\begin{array}{l}\text { Study } 3 \\
\text { (pilot for Study 4) }\end{array}$ & $11 / 0 \mathrm{HC}$ & $24.2 \pm 1.3$ & No medication & Probability & Abler et al. (2006) \\
\hline $\begin{array}{l}\text { Study } 4 \\
\text { (dopaminergic } \\
\text { manipulation) }\end{array}$ & 0/12 RLS & $55.8 \pm 8.8$ & $\begin{array}{l}\text { Patients scanned twice: } \\
\text { off-and-on their regular } \\
\text { dopaminergic medication }\end{array}$ & Probability & Abler et al. (2009) \\
\hline $\begin{array}{l}\text { Study } 5 \\
\text { (antidopaminergic } \\
\text { manipulation) }\end{array}$ & $4 / 4 \mathrm{HC}$ & $55.8 \pm 8.8$ & $\begin{array}{l}\text { Healthy controls scanned twice } \\
\text { under placebo and } \\
\text { after } 5 \text { mg olanzapine }\end{array}$ & Magnitude & Abler et al. (2008) \\
\hline
\end{tabular}

HC, Healthy controls; Sx, Patients with schizophrenia; RLS, Restless Legs Syndrome; SD, standard deviation.

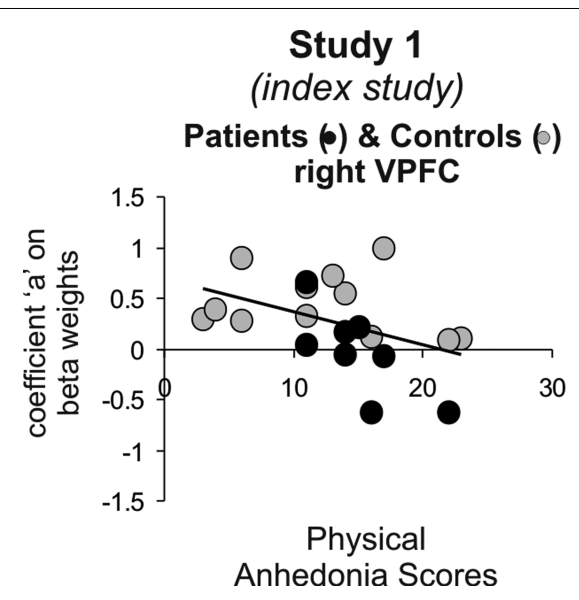

FIGURE 2 | SALIENCE and psychopathology (reward magnitude). In study 1 (index study) and study 2 (replication study) we found significant negative correlations with anhedonia scores (social anhedonia and physical anhedonia, respectively) and the coefficient " $a$ " extracted from the VLPFC where group differences (controls > patients with schizophrenia) were found, that is, lower anhedonia scores predicted a pattern with steeper U-shaped

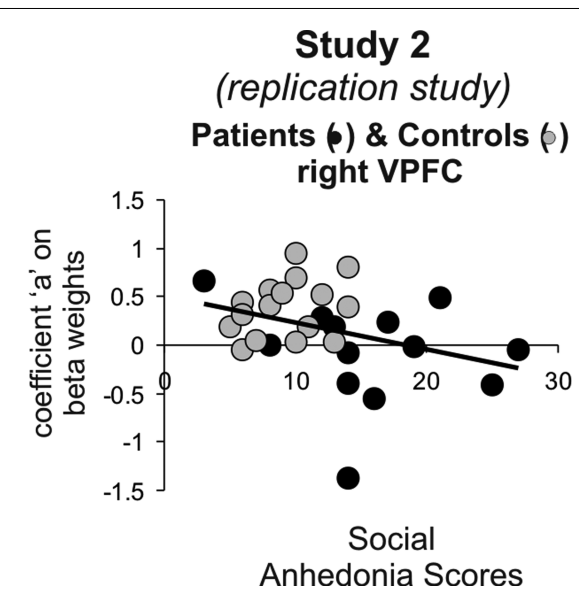

curves as found in the controls. Correlations were significant for the social anhedonia scores $(p=0.04)$ in all subjects, patients and controls in the index study 1 and for the physical anhedonia scores in study 2 in all subjects $(p=0.03)$ driven by a significant effect in the patients $(p=0.02)$. Anhedonia scores were not obtained in three of 16 patients in study 1 and 4 of 12 patients in study 2. 
descriptions of the respective sample characteristics are given in the papers referenced. All participants, patients and controls, gave written informed consent after complete description of the study. All studies were carried out in accordance with the latest version of the Declaration of Helsinki and approved by the ethics committee of the University of Ulm, Germany or, in case of the replication study by the ethics committee of McLean Hospital, Belmont, MA.

\section{RATINGS AND PSYCHOLOGICAL TESTING}

Psychopathological ratings refer to the German or English version of the Positive and Negative Syndrome Scale (PANSS, Kay et al., 1987; Müller, 2002) and the Physical and Social Anhedonia Scale (Chapman etal., 1976; Scherbarth-Roschmann and Hautzinger, 1991).

\section{REWARD TASKS}

Subjects in all studies were presented with one of two versions of a validated paradigm (Abler et al., 2005, 2006), that is, a monetary incentive task with a parametric variation of possible wins either regarding reward magnitude (Magnitude Paradigm: $1 €, 20 \$$, No win; probability of rewards fixed at $60 \%$ ) or reward probability (Probability Paradigm: 0\%, 25\%, 50\%, 75\%, 100\%, magnitude of rewards fixed at $1 €)$. Studies 1, 2 and 5 were conducted with the Probability Paradigm, studies 3 and 4 with the Magnitude Paradigm. In short, each of the 60 trials per session started with a symbol indicating one of three possible amounts of money to win or one of five probabilities. After an expectation period, subjects had to correctly react with a left or right button press to one of two symbols (a square or a triangle) within a fixed interval. Subjects were informed that they did not need to react faster and that their chances to win were independent of their reaction times. In reacting correctly in the Magnitude Paradigm, they preserved themselves a $60 \%$ chance to win the announced amount of money ( $1 €$ or $20 \$$ : win trial). In the Probability Paradigm, the announced chance to win $1 €$ was preserved. In the rest of the trials, subjects were not rewarded despite pressing the correct button (omission trials). Incorrect button presses resulted in a feedback of zero Euro at any rate. Win and omission trials as well as the trial types ( $1 €, 20 \notin$, no win/0\%, 25\%, 50\%, 75\%, 100\%) appeared in random order. In the control trials (no win/0\%) no money was announced, subjects only had to press an arbitrary button and could not win any money. To make sure that all trials included a button press of any kind, subjects were informed that they would lose $1 €$ if no button press occurred. Feedback (outcome) followed the targets disappearance and notified subjects with the amount of money they won in the trial.

\section{fMRI ACQUISITION}

Data for all studies were acquired on Siemens Scanners (Siemens AG, Germany): study 1, 3, 4 and 5 on a 3 Tesla ALLEGRA Scanner, study 2 on a 3 Tesla TRIO Scanner. Both scanners were equipped with a head coil and used to acquire $\mathrm{T} 1$ anatomical volume images $(1 \times 1 \times 1 \mathrm{~mm}$ voxels $)$ and functional MR images. Twenty-three axial slices (study 2: 21) were acquired with an image size of $64 \times 64$ pixels and a FoV of $192 \mathrm{~mm}$. Slice thickness was $3 \mathrm{~mm}$ with $0.75-\mathrm{mm}$ gap resulting in a voxel size of $3 \times 3 \times 3.75 \mathrm{~mm}$. Images were angled along a line connecting basal forebrain and basal cerebellum. Images were centred on basal structures of the brain including subcortical regions of interest (basal ganglia, orbitofrontal and ventral frontal regions). Functional images were recorded using a $\mathrm{T} 2{ }^{\star}$-sensitive gradient echo sequence measuring changes in BOLD-contrast. Four hundred and one volumes were obtained during each of the two reward sessions at a TR of $1500 \mathrm{~ms}$.

\section{fMRI ANALYSIS}

Image processing and statistical analysis were carried out using Statistical Parametric Mapping (SPM2, Wellcome Department, London, UK). To test for effects of reward salience, the respective contrast was calculated for each of the studies as depicted in Figures 1 and 2 and explained below in Section "Second level analyses".

\section{Preprocessing}

Images of all studies were pre-processed including slice timing, realignment to correct for motion artefacts and spatial normalization to standard space. Smoothing was applied with an $8-\mathrm{mm}$ Gaussian kernel. Intrinsic autocorrelations were accounted for by an autoregressive model of first order $(\mathrm{AR}(1))$ and low frequency drifts were removed via high pass filter.

\section{First level analysis}

After preprocessing, first level analysis was performed on each subject estimating the variance of voxels according to a general linear model for the Magnitude or Probability Paradigm: the three/five expectation periods, the button press and the five/eight different outcome events were modelled as boxcar functions and convolved with the hemodynamic response function. Depending on the preceding reward expectation (high/low/no or $0-100 \%$ ) and actual outcome (receipt of reward: $r$, omission of reward: $o$ ), the five/eight outcome events were: Magnitude Paradigm: R/high, R/low, no, O/low, O/high reward; Probability Paradigm: (i) 0\%; (ii) r/25\%; (iii) o/25\%; (iv) r/50\%; (v) o/50\%; (vi) r/75\%; (vii) o/75\%; and (viii) $100 \%$. The six realignment parameters were included in the models. Contrast images of contrasts between regressors of interest were calculated on the single subject level. This approach meant that new first level analyses with five instead of only three (receipt/omission/no reward) outcome regressors were calculated for studies 2 and 5, while the first level analyses of studies 1, 3 and 4 remained as published.

\section{Second level analyses}

Separately for each study, the contrast images of parameter estimates from the first level analysis were then included in a second level group analysis (random-effects model), treating inter-subject variability as a random effect to account for interindividual variance. We computed analyses on the five/eight outcome events (receipt/omission of reward). Effects were tested separately for each group (patients/control subjects/on and off medication) and by interaction analyses of the groups.

To model the salience of outcome events, we built a regular $\mathrm{U}$-shaped contrast as described in the index study: receipt and omission of high rewards were weighted with the highest contrast values; receipt and omission of low rewards were weighted with medium values, and the no reward events with the lowest contrast values. 
For the new analyses of the studies in this paper, thresholding was kept comparable to the respective original investigations. In the index study (study 1), whole brain random-effects statistical maps were thresholded at $p<0.001$ uncorrected for multiple comparisons at a cluster size threshold of 15 voxels. In studies 2, 4 and 5 a threshold of $p<0.001$ uncorrected at the voxel-level for simple contrasts and $p<0.005$ uncorrected for interaction contrasts at an extent threshold of $p<0.05$ at the cluster-level was used. If activations remained significant after FDR (false discovery rate) correction for multiple testing at $p<0.05$, they are indicated in Table 2 . In study 3 results are reported at a threshold of 0.005 family wise error (FWE) corrected. To ensure that the results of the interaction contrasts were clearly driven by actually elevated fMRI signal in one group, we computed the respective contrasts in the respective group only and used them as inclusive masks. Masks were thresholded at $p<0.005$ at the voxel-level.
Additional statistics on second level results. Regression analyses on the beta values of the peak voxels of activations found were calculated externally using the software packages Microsoft Excel and Statistica 6.0. The regression curve for a U-shaped contrast like our salience contrast is best described with a parabolic line that can be characterized by a second order equation $\left(a x^{2}+b x+c=y\right)$. The coefficient " $a$ " can be taken as a measure of the shape of the $U$ with lower values indicating a more shallow curve and negative values an inverse $U$-shape.

\section{Correlational analyses}

To assess relations between psychopathology and brain activation, we calculated planned correlations parallel to those in the index study between our main results concerning brain activation, that is, the brain regions where group differences were found and the main psychopathology scores, PANSS positive, negative, and anhedonia.

Table 2 | fMRI activations as found for the contrast modelling reward salience

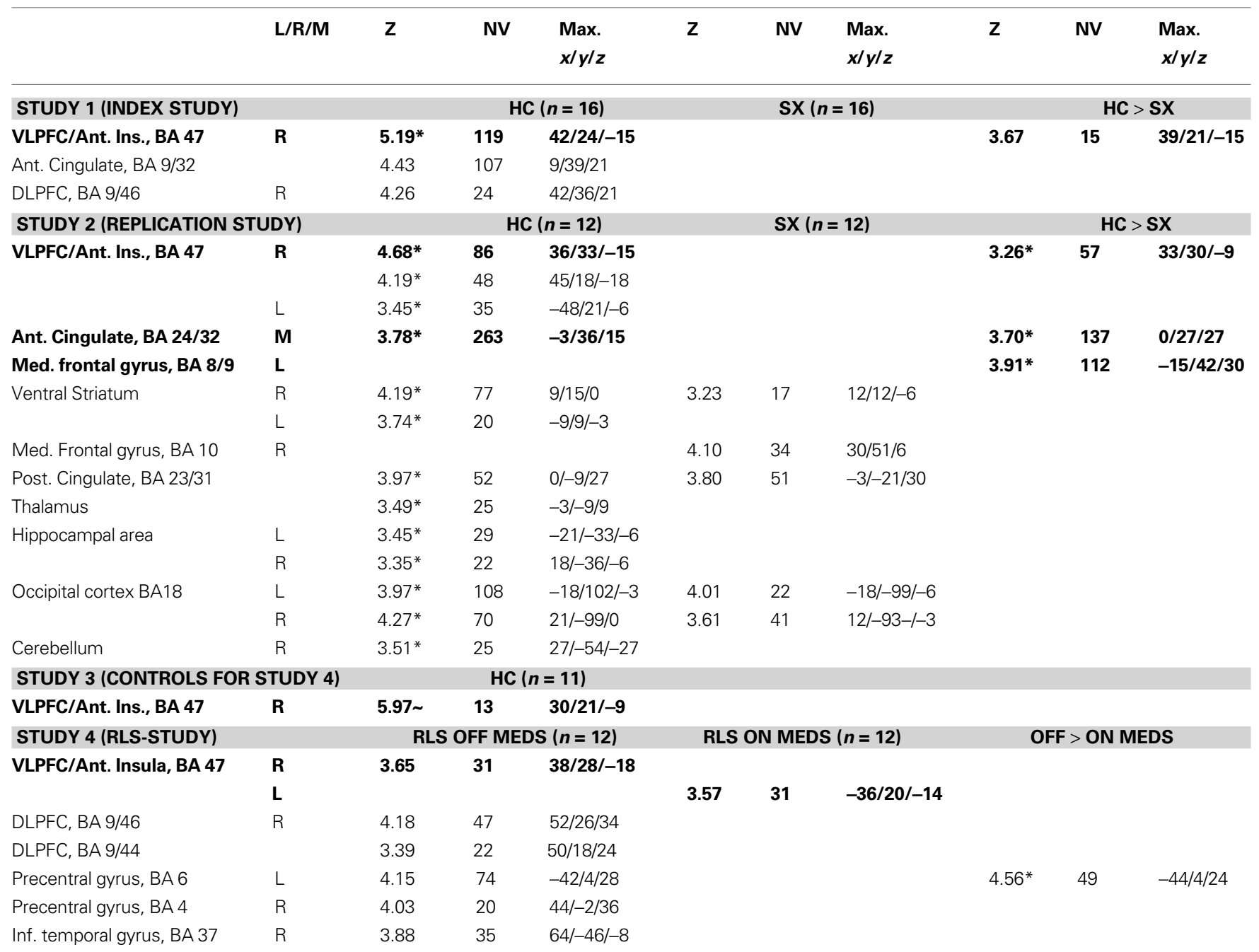




\section{RESULTS}

We investigated main effects of salience (controls, patients, on medication, off medication) and the interaction of salience by group (controls vs. patients, on vs. off medication) during the outcome phase.

\section{STUDY 1 (INDEX STUDY)}

Results from the salience contrast from the index study were taken directly from Walter et al. (2009).

\section{STUDY 2 (REPLICATION STUDY)}

Like in the index study, we found activation in the right VLPFC and adjacent anterior insula and anterior cingulate in controls but not in patients with schizophrenia. Replicating the result from the index study, the interaction contrast between healthy controls and patients diagnosed with schizophrenia showed differences in the VLPFC/anterior insula and furthermore the anterior cingulate cortex (Figure 2, Table 1).

The regression curve for the U-shaped contrast (Figure 2) in this study with the magnitude paradigm is ideally described with a parabolic line that can be characterized by the second order equation $\left(2 x^{2}-13 x+15=y\right)$ with the coefficient " $a=2$ " as a measure of the shape of the U. Parameter estimates were extracted from the peak voxel (33/30/-9) found for the interaction contrast that revealed the difference between healthy controls and patients with schizophrenia. Again, like in the index study, the values for the coefficient " $a$ " in the control subjects ( $a=0.45)$ were significantly higher than in the patients suffering from schizophrenia $(a=-0.07$; $t=3.39, p=0.003$ ).

\section{Correlational analyses in study 2 and study 1}

In the replication study 2 , we found a significant negative correlation $(r=-0.43, p=0.03)$ of the Physical Anhedonia scores in all subjects, patients and controls and the coefficient " $a$ " characterizing the regression curves, that is, lower anhedonia scores predicted a pattern with more U-shaped curves more similar to the controls (Figure 2). The correlation was driven by the significant correlation in the patients $(r=-0.75, p=0.02)$. In controls alone, the correlation was not significant $(r=-0.23, p>0.05[0.24])$. Likewise, in the index study 1 , we found a significant negative correlation $(r=-0.34$, $p=0.036)$ of the Social, but not Physical Anhedonia scores in all subjects, patients and controls and the coefficient " $a$ " characterizing the regression curves. However, this correlation was partly driven by the group differences in both, fMRI signal and anhedonia scores as can be seen in Figure 3.

Positive and Negative Syndrome Scale positive and negative scores did not correlate significantly in the index or the replication study.

\section{STUDY 3 (HEALTHY CONTROLS)}

In the control study for the probability paradigm, we found activation in the right VLPFC (Figure 1, Table 2) for the salience contrast.

The regression curve for the $\mathrm{U}$-shaped contrast in this study with the probability paradigm is ideally described with a parabolic line that can be characterized by the second order equation $\left(1 / 3 x^{2}-3 x+5=y\right)$ with the coefficient " $a=1 / 3$ " as a measure of the shape of the U. The value for the coefficient " $a$ " in this study was 0.21 .

\section{STUDY 4 (RLS-STUDY)}

In this study using the probability paradigm, we found activation in the right VLPFC only when patients were off their regular medication with dopamine agonists. The coefficient " $a$ " that can be taken as a measure of the shape of the $\mathrm{U}$ of this contrast (see Section "Study 2") had a value of $a=1.8$ in the peak voxel of this activation (ideal value $a=1 / 3$ for probability paradigm). The value of " $a$ " in this voxel when patients were on their regular medication was 0.02 . There was a trend for higher coefficients " $a$ " off medication $(t=1.7, p=0.058)$ although no significant interaction effect was found for the whole brain analysis in this region (Figure 3). On medication, the whole brain analysis revealed activation in the left VLPFC. However, inspection of the parameter estimates in this region revealed that this effect was mainly driven by highly positive values for the regressor $\mathrm{o} / 75 \%$ and to a lower extent by the $\mathrm{U}$-shape of the contrast which was quite far from ideal $(a=0.11)$.

\section{STUDY 5 (OLANZAPINE STUDY)}

We did not find any significant brain activation related to reward salience in the study comparing the effects of a single dose of olanzapine compared to placebo using the magnitude paradigm.

\section{DISCUSSION}

In this study, we investigated whether we could replicate our finding of aberrant salience coding in the right VLPFC observed in medicated patients with schizophrenia. Indeed, in an independent cohort of patients, the right VLPFC again showed a significant decrease in salience coding in the patients. In both studies, aberrant salience coding proved to be related to negative symptoms as evidenced by negative correlations with anhedonia, which is in line with the revised dopamine hypothesis. Finally, we found only modest evidence that dopaminergic stimulation in patients with RLS might impact on the representation of reward salience in the right VLPFC.

\section{ABERRANT SALIENCE CODING IN THE RIGHT VLPFC IN SCHIZOPHRENIA}

It is noteworthy that we were able to demonstrate the involvement of the right VLPFC in salience coding in now four independent cohorts, independently of the reward parameter, that is, for reward magnitude as well as reward probability. As in our index study, the right VLPFC showed significantly reduced activation in the salience contrast with correspondingly a significantly smaller coefficient " $a$ " (Figure 1) in patients. Although two other cortical regions, namely the anterior cingulate cortex as well as the left superior frontal cortex showed a similar pattern, we focus our discussion on the VLPFC as it was the only region common to both, study 1 and 2 .

The right VLPFC has been suggested to play a crucial role in the change of response strategies (Cools et al., 2002) as well as in response suppression (Arana et al., 2003) in the context of reward paradigms. Furthermore, the VLPC is involved in set shifts in problem-solving tasks (Goel and Vartanian, 2005). These functions are consistent with the hypothesized role of this brain region in salience processing according to the definition above: The experience of salient events, may they be positive or negative, is important to adapt future behavior. Therefore, 


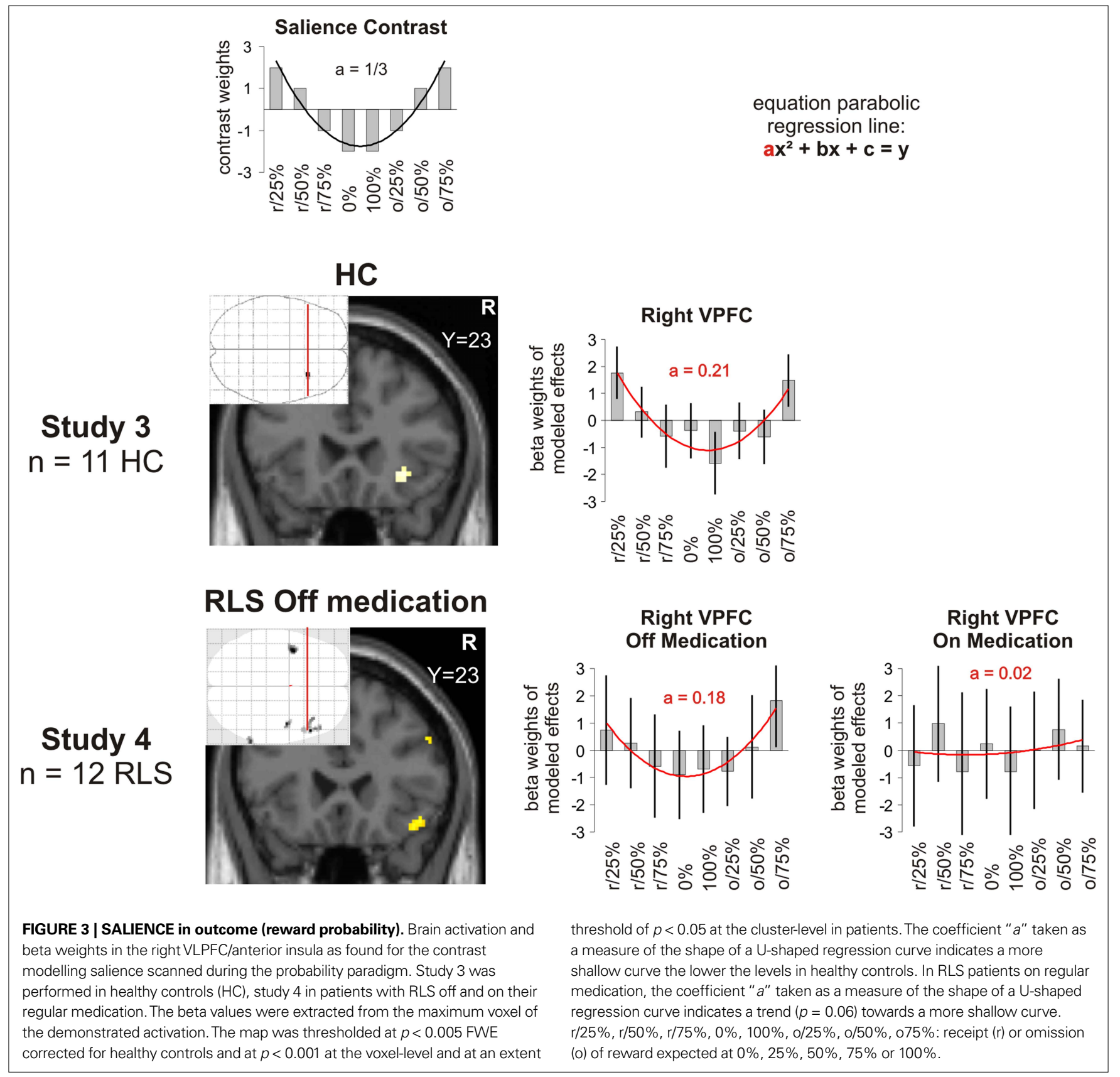

VLPFC hypoactivation to salient events may help to explain deficits in planning, decision making and action selection frequently observed in patients with psychosis. Accordingly, we would like to suggest that the dysfunctional activation of the right VLPFC related to salience processing found in our patients suffering from schizophrenia might reflect a deficit in transforming the salience signals into actions. This suggestion is further strengthened by the findings that an important dimension of negative symptoms, anhedonia, was linked to aberrant salience coding in both studies. This correlation is consistent with the hypothesized role of a reduced dopaminergic tone in the prefrontal cortex by the revised dopamine hypothesis. Study 1 found a correlation with social anhedonia, study 2 a

correlation with physical anhedonia. Thus it can be speculated that reduced dopaminergic tone contributes to different types of anhedonia.

Interestingly, concurrent with the aberrant salience hypothesis of psychosis, altered activation of the right VLPFC cortex was reliably found in a meta-analyses of fMRI studies on nonpsychotic relatives of patients with schizophrenia (Macdonald et al., 2008) where it was described as increased. A finding of increased regional activation in subjects at risk for schizophrenia in the face of usually decreased activation in patients with schizophrenia is found quite often and can be explained as a compensatory activation in subjects predisposed but not suffering from schizophrenia. Consistently, in patients actually suffering 
from schizophrenia deficits in tasks mediated by ventrolateral prefrontal brain regions like reversal learning paradigms (Waltz and Gold, 2007) or the Iowa Gambling Task (Ritter et al., 2004) have been repeatedly demonstrated.

\section{EFFECTS OF DOPAMINERGIC MANIPULATIONS ON PREFRONTAL SALIENCE CODING}

Although we could confirm our main hypothesis and replicate the results from our index study and found correlations with psychpathology according to our hypothesis, the results in the study using dopaminergic manipulation were not as clear as we had hoped. We could not demonstrate a decrease in salience coding by olanzapine. However, it should be noted that the olanzapine study (study 5) is also the only study in which salience coding in the VLPFC in healthy controls (here on placebo) could not be replicated. This might be due to the small number of subjects investigated $(n=8)$.

Concerning dopaminergic manipulation, the results are at least partially supporting an influence of dopamine on salience coding. Whereas RLS patients off medication showed salience coding in the right VLPFC, as well as in anterior cingulate cortex and DLPFC, these effects vanished when the patients were on medication. This could be explained by the fact that non-salient events are also coded as salient and that the right VLPFC therefore cannot properly distinguish between salient and non-salient stimuli.

However, it has to be noted that the group difference was not significant and that the change in the " $a$ " coefficient only showed a statistical trend. Moreover, an interpretation is limited by the fact that the subjects included in this study were patients with RLS, a disease that is generally accepted to be linked to central dopaminergic dysfunction, so that these effects cannot be interpreted in a straightforward manner. Also side effects of medication have to be taking into account. Reported side effects that could be related to altered salience processing are impaired impulse control and pathological gambling. Of our patients, none reported problems with impulse control or manifest pathological gambling, which was an exclusion criterion, although most patients had experienced nausea or dizziness (common side effects) when first receiving the medication. However, nausea and dizziness commonly wear off over the course of several days or weeks of regular medication.

\section{REFERENCES}

Abler, B., Erk, S., and Walter, H. (2007). Human reward system activation is modulated by a single dose of olanzapine in healthy subjects in an event-related, double-blind, placebo-controlled fMRI study. Psychopharmacology (Berl.) 191, 823-833.

Abler, B., Greenhouse, I., Ongur, D., Walter, H., and Heckers, S. (2008). Abnormal reward system activation in mania. Neuropsychopharmacology 33, 2217-2227.

Abler, B., Hahlbrock, R., Unrath, A., Gron, G., and Kassubek, J. (2009). At-risk for pathological gambling: imaging neural reward processing under chronic dopamine agonists. Brain 132, 2396-2402.
Abler, B., Walter, H., and Erk, S. (2005). Neural correlates of frustration. Neuroreport 16, 669-672.

Abler, B., Walter, H., Erk, S., Kammerer, H., and Spitzer, M. (2006). Prediction error as a linear function of reward probability is coded in human nucleus accumbens. Neuroimage 31, 790-795.

Arana, F. S., Parkinson, J. A., Hinton, E., Holland, A. J., Owen, A. M., and Roberts, A.C. (2003). Dissociable contributions of the human amygdala and orbitofrontal cortex to incentive motivation and goal selection. J. Neurosci. 23, 9632-9638.

Berridge, K.C., and Robinson, T.E. (2003). Parsing reward. Trends Neurosci. 26, 507-513.

Carlsson, A., and Lindquist, $M$. (1963). Effect of chlorpromazine

\section{LIMITATIONS}

Like in our index study, our results are limited by the fact that the patients with schizophrenia were treated with neuroleptics. For the ventral striatum it has been shown that atypical neuroleptics normalized the BOLD-response in comparison to untreated patients (Juckel et al., 2006a). Therefore, it could be the case that the results of aberrant salience coding are an effect of medication. Although we cannot exclude this possibility, it seems unlikely to us, as we did not only find categorical differences, but also a relation to psychopathology. Nevertheless, salience coding should be tested in non-medicated patients, an analysis that can easily be performed post hoc in studies already executed, a procedure we would like to encourage the respective groups to perform.

Furthermore, study 5 is limited not only by the small number of subjects but also by the fact that olanzapine is not a purely antidopaminergic drug but acts on a range of different neurotransmitters. As already mentioned, study 4 is limited by the fact that subjects are diagnosed having a disease impacting on central dopamine metabolism. Ideally, one would investigate a large group of healthy controls with one type of dopamine agonist, one type of a pure dopamine antagonist and a placebo.

\section{CONCLUSION}

In four of five studies we could replicate a significant activation of the right VLPFC for the processing of salience. Our main hypothesis concerning schizophrenia was confirmed: We could replicate the result of aberrant salience coding in the right VLPFC in an independent cohort of medicated patients with schizophrenia. Furthermore, the negative correlations with anhedonia scores provide evidence that aberrant salience coding is clinically relevant. An explanation of our replicated finding could be a low level of cortical dopamine, as postulated by the revised dopamine hypothesis. We suggest that future studies investigating reward in schizophrenia should, in addition to analysing prediction and prediction errors, pay more attention to salience coding.

\section{ACKNOWLEDGMENT}

Study 2 was supported by a personal grant to Birgit Abler from the DAAD (German Academic Exchange Service).

or haloperidol on formation of 3 methoxytyramine and normetanephrine in mouse brain. Acta Pharmacol. Toxicol. (Copenh) 20, 140-144.

Carlsson, A., Waters, N., Waters, S., and Carlsson, M. L. (2000). Network interactions in schizophrenia - therapeutic implications. Brain Res. Brain Res. Rev. 31, 342-349.

Chapman, L. J., Chapman, J. P., and Raulin, M. L. (1976). Scales for physical and social anhedonia. J. Abnorm Soc. Psychol. 85, 374-382.

Cools, R., Clark, L., Owen, A. M., and Robbins, T. W. (2002). Defining the neural mechanisms of probabilistic reversal learning using event-related functional magnetic resonance imaging. J. Neurosci. 22, 4563-4567.
Cooper, J. C., and Knutson, B. (2008). Valence and salience contribute to nucleus accumbens activation. Neuroimage 39, 538-547.

Corlett, P. R., Murray, G. K., Honey, G. D., Aitken, M. R., Shanks, D. R., Robbins, T. W., Bullmore, E. T. Dickinson, A., and Fletcher, P. C. (2007). Disrupted prediction-error signal in psychosis: evidence for an associative account of delusions. Brain 130, 2387-2400.

Goel, V., and Vartanian, O. (2005). Dissociating the roles of right ventral lateral and dorsal lateral prefrontal cortex in generation and maintenance of hypotheses in set-shift problems. Cereb. Cortex 15, 1170-1177.

Guillin,O.,Abi-Dargham,A., and Laruelle, M. (2007). Neurobiology of dopamine 
in schizophrenia. Int. Rev. Neurobiol. $78,1-39$.

Heinz, A. (2002). Dopaminergic dysfunction in alcoholism and schizophrenia-psychopathological and behavioral correlates. Eur. Psychiatry 17, 9-16.

Jensen, J., Smith, A. J., Willeit, M., Crawley, A. P., Mikulis, D. J., Vitcu, I., and Kapur, S. (2007). Separate brain regions code for salience vs. valence during reward prediction in humans. Hum. Brain Mapp. 28, 294-302.

Jensen, J., Willeit, M., Zipursky, R. B., Savina, I., Smith, A. J., Menon, M., Crawley,A.P., and Kapur,S. (2008). The formation of abnormal associations in schizophrenia: neural and behavioral evidence. Neuropsychopharmacology $33,473-479$.

Juckel, G., Schlagenhauf, F., Koslowski, M., Filonov, D., Wustenberg, T., Villringer, A., Knutson, B., Kienast, T., Gallinat, J., Wrase, J., and Heinz, A. (2006a). Dysfunction of ventral striatal reward prediction in schizophrenic patients treated with typical, not atypical, neuroleptics. Psychopharmacology (Berl.) 187, 222-228.

Juckel, G., Schlagenhauf, F., Koslowski, M., Wustenberg, T., Villringer, A., Knutson, B., Wrase, J., and Heinz, A. (2006b). Dysfunction of ventral striatal reward prediction in schizophrenia. Neuroimage 29, 409-416.

Kapur, S. (2003). Psychosis as a state of aberrant salience: a framework linking biology, phenomenology, and pharmacology in schizophrenia. Am. J. Psychiatry 160, 13-23.
Kapur, S., Agid, O., Mizrahi, R., and Li, M. (2006). How antipsychotics workfrom receptors to reality. NeuroRx 3 , $10-21$.

Kay, S. R., Fiszbein, A., and Opler, L. A. (1987). The positive and negative syndrome scale, PANSS for schizophrenia. Schizophr. Bull. 13, 261-276.

Knutson, B., and Cooper, J. C. (2005). Functional magnetic resonance imaging of reward prediction. Curr. Opin. Neurol. 18, 411-417.

Knutson, B., Fong, G. W., Adams, C. M., Varner, J. L., and Hommer, D. (2001). Dissociation of reward anticipation and outcome with event-related fMRI. Neuroreport 12, 3683-3687.

Macdonald, A. W., III, Thermenos, H. W., Barch, D. M., and Seidman, L. J. (2008). Imaging genetic liability to schizophrenia: systematic review of fMRI studies of patients' nonpsychotic relatives. Schizophr Bull. 35, 1142-1162.

Müller, M. J. (2002). Positive and Negative Syndrome Scale (PANSS). In: Diagnostische Verfahren in der Psychotherapie. Diagnostik für Klinik und Praxis, Band 1. E. J. Brähler, and B. Schumacher Strauß, eds (Hogrefe, Göttingen).

Murray, G. K., Corlett, P. R., Clark, L., Pessiglione, M., Blackwell, A. D., Honey, G., Jones, P. B., Bullmore, E. T., Robbins, T. W., and Fletcher, P. C. (2008). Substantia nigra/ventral tegmental reward prediction error disruption in psychosis. Mol. Psychiatry 13:239, 267-276.
Ritter, L. M., Meador-Woodruff,J.H., and Dalack, G. W. (2004). Neurocognitive measures of prefrontal cortical dysfunction in schizophrenia. Schizophr. Res. 68, 65-73.

Scherbarth-Roschmann, P., and Hautzinger, M. (1991). Zur psychometrischen Erfassung von Schizotypie: Methodische Überprüfung und erste Validierung von zwei Skalen zur Erfassung von Risikomerkmalen. Zeitschrift für Klinische Psychologie 20, 238-250.

Schlagenhauf, F., Sterzer, P., Schmack, K., Ballmaier, M., Rapp, M., Wrase, J., Juckel, G., Gallinat, J., and Heinz, A. (2009). Reward feedback alterations in unmedicated schizophrenia patients: relevance for delusions. Biol. Psychiatry 65, 1032-1039.

Spitzer, M. (1997). A cognitive neuroscience view of schizophrenic thought disorder. Schizophr. Bull. 23, 29-50.

Walter, H., Kammerer, H., Frasch, K. Spitzer,M., and Abler,B.(2009).Altered reward functions in patients on atypical antipsychotic medication in line with the revised dopamine hypothesis of schizophrenia. Psychopharmacology (Berl) 206, 121-132.

Waltz, J. A., and Gold, J. M. (2007) Probabilistic reversal learning impairments in schizophrenia: further evidence of orbitofrontal dysfunction. Schizophr. Res. 93, 296-303.

Wise, R. A., Spindler, J., deWit, H., and Gerberg, G. J. (1978). Neurolepticinduced "anhedonia" in rats: pimozide blocks reward quality of food. Science 201, 262-264.

Yacubian, J., Glascher, J., Schroeder, K., Sommer, T., Braus, D. F., and Buchel, C. (2006). Dissociable systems for gainand loss-related value predictions and errors of prediction in the human brain. J. Neurosci. 26, 9530-9537.

Conflict of Interest Statement: Karel Frasch has received speaker honoraria from Janssen-Cilag, travel payments from AstraZeneca, Janssen-Cilag and Eli-Lilly and holds shares of the pharmaceutical company Stada. Henrik Walter has received a speaker honorarium from Janssen-Cilag. The other authors declare that the research was conducted in the absence of any commercial or financial relationships that could be construed as a potential conflict of interest.

Received: 01 September 2009; paper pending published: 20 October 2009; accepted: 29 December 2009; published online: 09 February 2010.

Citation: Walter H, Heckers S, Kassubek J, Erk S, Frasch Kand Abler B (2010) Further evidencefor aberrant prefrontal salience coding in schizophrenia. Front. Behav. Neurosci. 3:62. doi: 10.3389/neuro.08.062.2009

Copyright () 2010 Walter, Heckers, Kassubek, Erk, Frasch and Abler. This is an open-access article subject to an exclusive license agreement between the authors and the Frontiers Research Foundation, which permits unrestricted use, distribution, and reproduction in any medium, provided the original authors and source are credited. 